\title{
Dynamic Design of Thick Orthotropic Cantilever Plates with Consideration of Bimoments
}

\author{
Makhamatali K. Usarov \\ Institute of Seismic Stability of Structures of the Academy of Sciences of the Republic of Uzbekistan, Tashkent, Uzbekistan \\ Email: umakhamatali@mail.ru
}

How to cite this paper: Usarov, M.K. (2016) Dynamic Design of Thick Orthotropic Cantilever Plates with Consideration of Bimoments. World Journal of Mechanics, 6, 341356.

http://dx.doi.org/10.4236/wjm.2016.610025

Received: September 6, 2016

Accepted: October 14, 2016

Published: October 17, 2016

Copyright $\odot 2016$ by author and Scientific Research Publishing Inc. This work is licensed under the Creative Commons Attribution International License (CC BY 4.0).

http://creativecommons.org/licenses/by/4.0/ (c) (i) Open Access

\begin{abstract}
The paper is devoted to dynamic design of thick orthotropic cantilever plates by applying the bimoment theory of plates, which takes into account the forces, moments and bimoments; and the theory takes into account nonlinear law of displacements distribution in cross section of the plate. The methods for constructing bimoment theory are based on Hooke's Law, three-dimensional equations of the theory of dynamic elasticity and the method of displacements expansion into Maclaurin series. The article gives the expressions to determine the forces, moments and bimoments. Bimoment theory of plates is described by two unrelated two-dimensional systems with nine equations in each. On each edge of the plate, depending on the type of fastening, nine boundary conditions are given. As an example, the solution of the problem of dynamic bending of thick isotropic and orthotropic plate under the influence of transverse dynamic loads in the form of the Heaviside function is given. The equations of motion of the plate are solved by numerical method of finite differences. The numerical results are obtained for isotropic and orthotropic plate. The graphs of changes of displacements and stresses of faces surfaces of the plate are presented. Maximum values of these displacements are found and analyzed. It is shown that by Timoshenko theory numerical values of stresses are much smaller compared to the ones obtained by bimoment theory of plates. Maximum numerical values of generalized displacements, forces, moments, and bimoments are obtained and presented in tabular form. The analysis of numerical results is done and the conclusions are drawn.
\end{abstract}

\section{Keywords}

Hooke's Law, Thick Plate, Dynamic Theory of Elasticity, Three-Dimensional Problem, Bimoment Theory

\section{Introduction}

The theory and the methods of thick plate design are developed as an applied part of 
the Mechanics of rigid body. Existing theories of thick plates considering transverse shear of plates are based on a number of simplifying hypotheses proposed by many researchers. There are numerous papers and monographs of Russian and foreign authors in this direction. Literature review on the theory and design of plates within the specified theory is given in [1]-[4].

Static problems of bending of thick isotropic plates within the three-dimensional theory of elasticity are considered in [5] (B. F. Vlasov); it gives an exact analytical solution in trigonometric series. The monograph by E. N. Baida [6] throws light upon the question of bending of orthotropic plates in trigonometric series. Numerical results of displacements and stresses are obtained.

In recent years, a number of studies have been published on static and dynamic analysis of structural elements in the field of the theory of plates. The authors [7] are involved in dynamic tasks of anisotropic plate vibrations. Foreign authors Karamooz Ravari M. R. and Forouzan M. R. [8] have considered the problem of free oscillations of a circular ring orthotropic plate. Frequency equations have been built in vibration plane for general boundary conditions.

The work of the authors in [9] is devoted to solving the problem of transient oscillations of a rectangular viscoelastic orthotropic plate on the basis of Flügge and Timoshenko-Mindlin deformation models. The paper [10] solves the problem of steady forced oscillations of orthotropic plate by superposition method, which is reduced to a quasi-regular infinite system of linear equations; its analytical solution is built. In [11] on the basis of the method of separation of variables a three-dimensional problem of elasticity theory is solved. The method of design of rectangular orthotropic elastic plates subjected to external loads on the upper and lower faces is developed

Papers [12] [13] are devoted to the construction of the theory of plate by displacements expansion into a series on one of the spatial coordinates oriented along the normal of the plate. Displacements in the plate plane can be expanded in the form of a cubic parabola, and normal displacements-in the form of a quadratic parabola. In [12], a problem of plate bending is solved and a comparative analysis with the results of other authors is carried out. In [13], dynamic bending of thick rectangular plate under the action of lumped dynamic forces is considered. Numerical results are obtained.

If to consider the law of nonlinearity of displacements distribution in the crosssections of the plate, then in addition to tensile and shear forces, bending and torsional moments, there appear the additional force factors, called the bimoments. In [14]-[17] the development and solution of the problem of bending and vibrations of thick plates is based on bimoment theory of plates built within the three-dimensional theory of elasticity without simplifying hypotheses, using the method of displacements expansion into Maclaurin infinite series on one of the spatial coordinates.

This paper is dedicated to dynamic analysis of thick plates on the basis of bimoment theory of plates. To take into account all force factors of the plate, including the bimoments, one should consider all the components of stress and strain tensors: $\sigma_{i j}, \varepsilon_{i j},(i, j=1,3)$. The components of displacement vector are presented in the form of 
a function of three spatial coordinates and time

$u_{1}\left(x_{1}, x_{2}, z, t\right), u_{2}\left(x_{1}, x_{2}, z, t\right), u_{3}\left(x_{1}, x_{2}, z, t\right)$.

The statements of dynamic problem for thick plates in three-dimensional formulation and the methods of reducing it to a two-dimensional bimoment theory are briefly described. Determinant correlations of forces, moments, and bimoments, as well as the equations of motion of the plate, given in [14]-[16] are produced relative to these force factors.

\section{Statement of the Problem}

Consider an orthotropic thick plate of constant thickness $H=2 h$ and plan dimensions $a, b$. Introduce the denotations: $E_{1}, E_{2}, E_{3}$-elasticity modulus; $G_{12}, G_{13}, G_{23}$-shear modulus; $v_{12}, v_{13}, v_{23}$-Poisson ratio of material of the plate.

To describe the motion of the plate a Cartesian system of coordinates with variables $X_{1}, X_{2}$ and $Z$ is introduced. The origin is taken in the mid-surface of the plate. OZ axe is directed down.

Let the distributed surface, normal and tangent loads be applied to two face surfaces of the plate $z=-h$ and $z=h$. Normal loads $q_{3}^{(+)}, q_{3}^{(-)}$are applied along $O Z$ axe. Tangent loads $q_{k}^{(+)}, q_{k}^{(-)},(k=\overline{1,2})$ are applied in $O X_{1}, O X_{2}$ axes.

The plate is considered as a three-dimensional body, its material obeying the Hooke's generalized Law. Three-dimensional equations of dynamic theory of elasticity are used as an equation of motion of the plate.

Boundary conditions of face surfaces of the plate $z=h$ and $z=-h$ have the form:

$$
\begin{gathered}
\sigma_{33}=q_{3}^{(+)}, \sigma_{31}=q_{1}^{(+)}, \sigma_{32}=q_{2}^{(+)} \text {at } z=h, \\
\sigma_{33}=q_{3}^{(-)}, \quad \sigma_{31}=q_{1}^{(-)}, \sigma_{32}=q_{2}^{(-)} \quad \text { at } z=-h .
\end{gathered}
$$

\section{Method of Solution}

The methods of construction of bimoment theory of plates are based on Hooke's generalized Law, three-dimensional theory of elasticity, boundary conditions of face surfaces (1) and displacements expansion into Maclaurin series in the form:

$$
\begin{gathered}
u_{k}=B_{0}^{(k)}+B_{1}^{(k)} \frac{z}{h}+B_{2}^{(k)}\left(\frac{z}{h}\right)^{2}+B_{3}^{(k)}\left(\frac{z}{h}\right)^{3}+\cdots+B_{i}^{(k)}\left(\frac{z}{h}\right)^{i}+\cdots, \quad(k=1,2), \\
u_{3}=A_{0}+A_{1} \frac{z}{h}+A_{2}\left(\frac{z}{h}\right)^{2}+A_{3}\left(\frac{z}{h}\right)^{3}+\cdots+A_{i}\left(\frac{z}{h}\right)^{i}+\cdots
\end{gathered}
$$

where $B_{i}^{(k)}, A_{i}$-are unknown functions of two spatial coordinates

$$
\begin{aligned}
B_{i}^{(k)}=B_{i}^{(k)}\left(x_{1}, x_{2}, t\right), A_{i} & =A_{i}\left(x_{1}, x_{2}, t\right): \\
B_{i}^{(k)}= & \frac{1}{i !} h^{i}\left(\frac{\partial^{i} u_{k}}{\partial z^{i}}\right)_{z=0},(k=1,2), A_{i}=\frac{1}{i !} h^{i}\left(\frac{\partial^{i} u_{3}}{\partial z^{i}}\right)_{z=0} .
\end{aligned}
$$

Displacements of the points of face surfaces $z=-h$ and $z=+h$ of the plate are denoted by $u_{i}^{(-)}, u_{i}^{(+)},(i=1,3)$, and stresses on face surfaces $z=-h$ and $z=+h$ by $\sigma_{11}^{(-)}, \sigma_{12}^{(-)}, \sigma_{22}^{(-)}$and $\sigma_{11}^{(+)}, \sigma_{12}^{(+)}, \sigma_{22}^{(+)}$. 
Note that bimoment theory of plates is described by two unrelated problems, each of which is formulated on the basis of nine two-dimensional equations with appropriate boundary conditions. Determinant equations and equations of motion of bimoment theory of plates are briefly described.

The first problem consists of two equations for longitudinal and tangential forces and four subsidiary built equations for bimoments for the nine unknown kinematic functions:

$$
\begin{gathered}
\bar{u}_{k}=\frac{u_{k}^{(+)}+u_{k}^{(-)}}{2}, \bar{\psi}_{k}=\frac{1}{2 h} \int_{-h}^{h} u_{k} \mathrm{~d} z, \quad \bar{\beta}_{k}=\frac{1}{2 h^{3}} \int_{-h}^{h} u_{k} z^{2} \mathrm{~d} z, \quad(k=1,2) \\
\bar{W}=\frac{u_{3}^{(+)}-u_{3}^{(-)}}{2}, \quad \bar{r}=\frac{1}{2 h^{2}} \int_{-h}^{h} u_{3} z \mathrm{~d} z, \quad \bar{\gamma}=\frac{1}{2 h^{4}} \int_{-h}^{h} u_{3} z^{3} \mathrm{~d} z .
\end{gathered}
$$

Introduce load terms to the equation of motion for the first problem, $\bar{q}_{k},(k=1,2), \bar{q}_{3}$-are determined by formulae:

$$
\bar{q}_{k}=\frac{q_{k}^{(+)}-q_{k}^{(-)}}{2},(k=1,2), \quad \bar{q}_{3}=\frac{q_{3}^{(+)}+q_{3}^{(-)}}{2} .
$$

The forces $N_{11}, N_{12}, N_{22}$ and bimoments $T_{11}, T_{22}, T_{12}$ are determined by the expressions:

$$
\begin{gathered}
N_{11}=E_{11} H \frac{\partial \bar{\psi}_{1}}{\partial x_{1}}+E_{12} H \frac{\partial \bar{\psi}_{2}}{\partial x_{2}}+2 E_{13} \bar{W}, N_{22}=E_{12} H \frac{\partial \bar{\psi}_{1}}{\partial x_{1}}+E_{22} H \frac{\partial \bar{\psi}_{2}}{\partial x_{2}}+2 E_{23} \bar{W} \\
T_{11}=H\left(E_{11} \frac{\partial \bar{\beta}_{1}}{\partial x_{1}}+E_{12} \frac{\partial \bar{\beta}_{2}}{\partial x_{2}}+E_{13} \frac{2 \bar{W}-4 \bar{r}}{H}\right), T_{22}=H\left(E_{12} \frac{\partial \bar{\beta}_{1}}{\partial x_{1}}+E_{22} \frac{\partial \bar{\beta}_{2}}{\partial x_{2}}+E_{23} \frac{2 \bar{W}-4 \bar{r}}{H}\right)(6) \\
N_{12}=N_{21}=G_{12}\left(H \frac{\partial \bar{\psi}_{1}}{\partial x_{2}}+H \frac{\partial \bar{\psi}_{2}}{\partial x_{1}}\right), T_{12}=T_{21}=H G_{12}\left(\frac{\partial \bar{\beta}_{2}}{\partial x_{1}}+\frac{\partial \bar{\beta}_{1}}{\partial x_{2}}\right)
\end{gathered}
$$

The intensities of transverse bimoments $\bar{p}_{13}, \bar{p}_{23}$ and $\bar{\tau}_{13}, \bar{\tau}_{23}$ from tangential stresses $\sigma_{13}, \sigma_{23}$ have the form:

$$
\bar{p}_{k 3}=G_{k 3}\left(\frac{\partial \bar{r}}{\partial x_{k}}+\frac{2\left(\bar{u}_{k}-\bar{\psi}_{k}\right)}{H}\right), \bar{\tau}_{k 3}=G_{k 3}\left(\frac{\partial \bar{\gamma}}{\partial x_{k}}+\frac{2\left(\bar{u}_{k}-3 \bar{\beta}_{k}\right)}{H}\right), \quad(k=1,2) .
$$

And the intensities of normal bimoments $\bar{p}_{33}$ and $\bar{\tau}_{33}$ from normal stress $\sigma_{33}$ are determined by the formula:

$$
\bar{p}_{33}=E_{31} \frac{\partial \bar{\psi}_{1}}{\partial x_{1}}+E_{32} \frac{\partial \bar{\psi}_{2}}{\partial x_{2}}+E_{33} \frac{2 \bar{W}}{H}, \quad \bar{\tau}_{33}=E_{31} \frac{\partial \bar{\beta}_{1}}{\partial x_{1}}+E_{32} \frac{\partial \bar{\beta}_{2}}{\partial x_{2}}+E_{33} \frac{2 \bar{W}-4 \bar{r}}{H} .
$$

The equation of motion relative to longitudinal and tangential forces, acting in the plane of the plate, has the form:

$$
\begin{aligned}
& \frac{\partial N_{11}}{\partial x_{1}}+\frac{\partial N_{12}}{\partial x_{2}}+2 \bar{q}_{1}=\rho H \ddot{\bar{\psi}}_{1} \\
& \frac{\partial N_{21}}{\partial x_{1}}+\frac{\partial N_{22}}{\partial x_{2}}+2 \bar{q}_{2}=\rho H \ddot{\bar{\psi}}_{2}
\end{aligned}
$$


As could be seen, the systems of two Equation (10) contains three unknown functions $\bar{\psi}_{1}, \bar{\psi}_{2}, \bar{W}$. To complete this system two equations of motion relative to longitudinal and tangential bimoments are written

$$
\begin{aligned}
& \frac{\partial T_{11}}{\partial x_{1}}+\frac{\partial T_{12}}{\partial x_{2}}-4 \bar{p}_{13}+2 \bar{q}_{1}=\rho H \ddot{\overline{\beta_{1}}} \\
& \frac{\partial T_{12}}{\partial x_{1}}+\frac{\partial T_{22}}{\partial x_{2}}-4 \bar{p}_{23}+2 \bar{q}_{2}=\rho H \ddot{\overline{\beta_{2}}}
\end{aligned}
$$

and two more equations of motion relative to the intensity of transverse bimoments in the following form:

$$
\begin{aligned}
& \frac{\partial \bar{p}_{13}}{\partial x_{1}}+\frac{\partial \bar{p}_{23}}{\partial x_{2}}-\frac{2 \bar{p}_{33}}{H}+\frac{2 \bar{q}_{3}}{H}=\rho \ddot{\bar{r}} \\
& \frac{\partial \bar{\tau}_{13}}{\partial x_{1}}+\frac{\partial \bar{\tau}_{23}}{\partial x_{2}}-\frac{6 \bar{\tau}_{33}}{H}+\frac{2 \bar{q}_{3}}{H}=\rho \ddot{\bar{\gamma}} .
\end{aligned}
$$

Using Maclaurin series (2) and the correlations (3), boundary conditions (1) are presented in the form of the system of three equations

$$
\begin{gathered}
\bar{u}_{k}=\frac{1}{4}\left(21 \bar{\beta}_{k}-3 \bar{\psi}_{k}\right)-\frac{1}{20} H \frac{\partial \bar{W}}{\partial x_{k}}+\frac{1}{20} \frac{H \bar{q}_{k}}{G_{k 3}}, \quad(k=1,2) \\
\bar{W}=\frac{1}{2}(21 \bar{\gamma}-7 \bar{r})-\frac{1}{30} H\left(\frac{E_{31}}{E_{33}} \frac{\partial \bar{u}_{1}}{\partial x_{1}}+\frac{E_{32}}{E_{33}} \frac{\partial \bar{u}_{2}}{\partial x_{2}}\right)+\frac{H \bar{q}_{3}}{30 E_{33}} .
\end{gathered}
$$

Equations of motion (10) - (15) comprise a combined system of differential equations from nine equations on unknown functions: $\bar{\psi}_{1}, \bar{\psi}_{2}, \bar{\beta}_{1}, \bar{\beta}_{1}, \bar{u}_{1}, \bar{u}_{2}, \bar{r}, \bar{\gamma}, \bar{W}$.

Note that all formulae of force factors (5) - (9) and equations of motion of the plate of the first problem (10) - (13) are strictly built. Approximation exists in derivation of the Equation (14) and Equation (15) only. Equation (14) is built with the fourth order of accuracy, and Equation (15) with the sixth order of accuracy relative to small parameter of the plate $\delta=\frac{H}{10 a}$. Here a-is a small size in plate plan.

The second problem consists in equations for bending moments, torsional moments, shear forces and bimoments relative to nine unknown kinematic functions:

$$
\begin{gathered}
\tilde{W}=\frac{u_{3}^{(+)}+u_{3}^{(-)}}{2}, \quad \tilde{r}=\frac{1}{2 h} \int_{-h}^{h} u_{3} \mathrm{~d} z, \quad \tilde{\gamma}=\frac{1}{2 h^{3}} \int_{-h}^{h} u_{3} z^{2} \mathrm{~d} z, \\
\tilde{u}_{k}=\frac{u_{k}^{(+)}-u_{k}^{(-)}}{2}, \quad \tilde{\psi}_{k}=\frac{1}{2 h^{2}} \int_{-h}^{h} u_{k} z \mathrm{~d} z, \quad \tilde{\beta}_{k}=\frac{1}{2 h^{4}} \int_{-h}^{h} u_{k} z^{3} \mathrm{~d} z, \quad(k=1,2) .
\end{gathered}
$$

Load terms of the second problem equation $\tilde{q}_{k},(k=1,2), \tilde{q}_{3}$ are determined in the following form:

$$
\tilde{q}_{k}=\frac{q_{k}^{+}+q_{k}^{-}}{2},(k=1,2), \quad \tilde{q}_{3}=\frac{q_{3}^{+}-q_{3}^{-}}{2} .
$$

Bending and torsional moments $M_{11}, M_{22}, M_{12}$ and $P_{11}, P_{22}, P_{12}$ are written as follows 


$$
\begin{gathered}
M_{11}=\frac{H^{2}}{2}\left(E_{11} \frac{\partial \tilde{\psi}_{1}}{\partial x_{1}}+E_{12} \frac{\partial \tilde{\psi}_{2}}{\partial x_{2}}-E_{13} \frac{2(\tilde{r}-\tilde{W})}{H}\right), \\
M_{22}=\frac{H^{2}}{2}\left(E_{12} \frac{\partial \tilde{\psi}_{1}}{\partial x_{1}}+E_{22} \frac{\partial \tilde{\psi}_{2}}{\partial x_{2}}-E_{23} \frac{2(\tilde{r}-\tilde{W})}{H}\right) \\
P_{11}=\frac{H^{2}}{2}\left(E_{11} \frac{\partial \tilde{\beta}_{1}}{\partial x_{1}}+E_{12} \frac{\partial \tilde{\beta}_{2}}{\partial x_{2}}-E_{13} \frac{2(3 \tilde{\gamma}-\tilde{W})}{H}\right), \\
P_{22}=\frac{H^{2}}{2}\left(E_{12} \frac{\partial \tilde{\beta}_{1}}{\partial x_{1}}+E_{22} \frac{\partial \tilde{\beta}_{2}}{\partial x_{2}}-E_{23} \frac{2(3 \tilde{\gamma}-\tilde{W})}{H}\right) \\
M_{12}=M_{21}=G_{12} \frac{H^{2}}{2}\left(\frac{\partial \tilde{\psi}_{1}}{\partial x_{2}}+\frac{\partial \tilde{\psi}_{2}}{\partial x_{1}}\right), \\
P_{12}=P_{21}=\frac{H^{2}}{2} G_{12}\left(\frac{\partial \tilde{\beta}_{1}}{\partial x_{2}}+\frac{\partial \tilde{\beta}_{2}}{\partial x_{1}}\right) .
\end{gathered}
$$

Expressions to define shear forces have the form:

$$
Q_{13}=G_{13}\left(2 \tilde{u}_{1}+H \frac{\partial \tilde{r}}{\partial x_{1}}\right), \quad Q_{23}=G_{23}\left(2 \tilde{u}_{2}+H \frac{\partial \tilde{r}}{\partial x_{2}}\right) .
$$

The intensity of transverse and normal bimoments $\tilde{p}_{13}, \tilde{p}_{23}$ and $\tilde{p}_{33}$ are determined by the expressions

$$
\tilde{p}_{k 3}=G_{k 3}\left(\frac{2 \tilde{u}_{k}-4 \tilde{\psi}_{k}}{H}+\frac{\partial \tilde{\gamma}}{\partial x_{k}}\right),(k=1,2), \quad \tilde{p}_{33}=E_{31} \frac{\partial \tilde{\psi}_{1}}{\partial x_{1}}+E_{32} \frac{\partial \tilde{\psi}_{2}}{\partial x_{2}}-E_{33} \frac{2(\tilde{r}-\tilde{W})}{H} .
$$

Equations of motion of the second problem are also described by a system of six equations of motion of the plate. The first three equations of motion are written for bending and torsional moments and one equation-for shear forces:

$$
\begin{gathered}
\frac{\partial M_{11}}{\partial x_{1}}+\frac{\partial M_{12}}{\partial x_{2}}-Q_{13}+H \tilde{q}_{1}=\frac{H^{2}}{2} \rho \ddot{\tilde{\psi}}_{1}, \\
\frac{\partial M_{21}}{\partial x_{1}}+\frac{\partial M_{22}}{\partial x_{2}}-Q_{23}+H \tilde{q}_{2}=\frac{H^{2}}{2} \rho \ddot{\tilde{\psi}}_{2}, \\
\frac{\partial Q_{13}}{\partial x_{1}}+\frac{\partial Q_{23}}{\partial x_{2}}+2 \tilde{q}_{3}=\rho H \ddot{\tilde{r}} .
\end{gathered}
$$

Three more equations of motion of the plate would be written for bimoments; two of them for bending and torsional bimoments have the form:

$$
\begin{aligned}
& \frac{\partial P_{11}}{\partial x_{1}}+\frac{\partial P_{12}}{\partial x_{2}}-3 H \tilde{p}_{13}+H \tilde{q}_{1}=\frac{H^{2}}{2} \rho \ddot{\tilde{\beta}}_{1} \\
& \frac{\partial P_{21}}{\partial x_{1}}+\frac{\partial P_{22}}{\partial x_{2}}-3 H \tilde{p}_{23}+H \tilde{q}_{2}=\frac{H^{2}}{2} \rho \ddot{\tilde{\beta}}_{2} .
\end{aligned}
$$

The sixth equation of plate motion for the intensity of transverse bimoments is written as follows: 


$$
H \frac{\partial \tilde{p}_{13}}{\partial x_{1}}+H \frac{\partial \tilde{p}_{23}}{\partial x_{2}}-4 \tilde{p}_{33}+2 \tilde{q}_{3}=H \rho \ddot{\tilde{\gamma}}
$$

Using Maclaurin series (2) and relationships (16), boundary conditions (1) are presented in the form of the system of three equations, written as:

$$
\begin{gathered}
\tilde{u}_{k}=\frac{1}{2}\left(21 \tilde{\beta}_{k}-7 \tilde{\psi}_{k}\right)-\frac{1}{30} H \frac{\partial \tilde{W}}{\partial x_{k}}+\frac{1}{30} \frac{H \tilde{q}_{k}}{G_{k 3}}, \quad(k=1,2), \\
\tilde{W}=\frac{1}{4}(21 \tilde{\gamma}-3 \tilde{r})-\frac{1}{20} H\left(\frac{E_{31}}{E_{33}} \frac{\partial \tilde{u}_{1}}{\partial x_{1}}+\frac{E_{32}}{E_{33}} \frac{\partial \tilde{u}_{2}}{\partial x_{2}}\right)+\frac{H \tilde{q}_{3}}{20 E_{33}} .
\end{gathered}
$$

The system of differential equations of motion (23) - (28) comprises a combined system of nine equations relative to nine unknown functions $\tilde{\psi}_{1}, \tilde{\psi}_{2}, \tilde{u}_{1}, \tilde{u}_{2}, \tilde{\beta}_{1}, \tilde{\beta}_{1}, \tilde{r}, \tilde{\gamma}, \tilde{W}$.

It should be noted that all formulae of force factors (18) - (22) and equations of motion of the plate for the second problem (23) - (26) are strictly built. Approximation exists in derivation of Equation (27) and Equation (28) only. Equation (28) is built with the fourth order of accuracy, and Equation (27) - with the sixth order of accuracy relative to small parameter of the plate $\delta$.

The stresses on the upper and lower face surfaces $z=-h$ and $z=+h$ are denoted by $\sigma_{11}^{(-)}, \sigma_{12}^{(-)}, \sigma_{22}^{(-)}$and $\sigma_{11}^{(+)}, \sigma_{12}^{(+)}, \sigma_{22}^{(+)}$. Using these expressions, one would introduce the force factors $\bar{\sigma}_{11}, \bar{\sigma}_{22}, \bar{\sigma}_{12}$ and $\tilde{\sigma}_{11}, \tilde{\sigma}_{22}, \tilde{\sigma}_{12}$, defined by formula:

$$
\bar{\sigma}_{i j}=\frac{\sigma_{i j}^{(+)}+\sigma_{i j}^{(-)}}{2}, \quad \tilde{\sigma}_{i j}=\frac{\sigma_{i j}^{(+)}-\sigma_{i j}^{(-)}}{2}, \quad(i=1,2 ; j=1,2) .
$$

The values $\bar{\sigma}_{11}, \bar{\sigma}_{22}, \bar{\sigma}_{12}$ and $\tilde{\sigma}_{11}, \tilde{\sigma}_{22}, \tilde{\sigma}_{12}$ are referred as bimoment intensities under tension-compression with consideration of transverse reduction and lateral bending with cross shear of the plate.

The intensities of the bimoments $\bar{\sigma}_{11}^{*}, \bar{\sigma}_{22}^{*}$ and $\tilde{\sigma}_{11}^{*}, \tilde{\sigma}_{22}^{*}$ are introduced by the differences and sums of derivatives in $z$-coordinate from normal stresses $\sigma_{11}, \sigma_{22}$ :

$$
\begin{aligned}
& \bar{\sigma}_{11}^{*}=\frac{H}{2}\left[\left(\frac{\partial \sigma_{11}}{\partial z}\right)_{z=+h}-\left(\frac{\partial \sigma_{11}}{\partial z}\right)_{z=-h}\right], \quad \bar{\sigma}_{22}^{*}=\frac{H}{2}\left[\left(\frac{\partial \sigma_{22}}{\partial z}\right)_{z=+h}-\left(\frac{\partial \sigma_{22}}{\partial z}\right)_{z=-h}\right], \\
& \tilde{\sigma}_{11}^{*}=\frac{H}{2}\left[\left(\frac{\partial \sigma_{11}}{\partial z}\right)_{z=+h}+\left(\frac{\partial \sigma_{11}}{\partial z}\right)_{z=-h}\right], \quad \tilde{\sigma}_{22}^{*}=\frac{H}{2}\left[\left(\frac{\partial \sigma_{22}}{\partial z}\right)_{z=+h}+\left(\frac{\partial \sigma_{22}}{\partial z}\right)_{z=-h}\right] .
\end{aligned}
$$

On the basis of Hooke's Law and boundary conditions (1.a) and (1.b) the expressions for half-difference and half-sum of the first derived functions $u_{1}, u_{2}$ and $u_{3}$ are found in $z$-coordinate on face surfaces of the plate $z=-h$ and $z=+h$

$$
\begin{gathered}
\left(\frac{\partial u_{k}}{\partial z}\right)_{z=+h}-\left(\frac{\partial u_{k}}{\partial z}\right)_{z=-h}=\frac{\bar{q}_{k}}{G_{k 3}}-\frac{\partial \bar{W}}{\partial x_{k}}, \quad(k=1,2) \\
\left(\frac{\partial u_{3}}{\partial z}\right)_{z=+h}+\left(\frac{\partial u_{3}}{\partial z}\right)_{z=-h}=\frac{1}{E_{33}}\left(\bar{q}_{3}-E_{31} \frac{\partial \bar{u}_{1}}{\partial x_{1}}-E_{32} \frac{\partial \vec{u}_{2}}{\partial x_{2}}\right), \\
\left(\frac{\partial u_{k}}{\partial z}\right)_{z=+h}+\left(\frac{\partial u_{k}}{\partial z}\right)_{z=-h}=\frac{\tilde{q}_{k}}{G_{k 3}}-\frac{\partial \tilde{W}}{\partial x_{k}}, \quad(k=1,2),
\end{gathered}
$$




$$
\left(\frac{\partial u_{3}}{\partial z}\right)_{z=+h}-\left(\frac{\partial u_{3}}{\partial z}\right)_{z=-h}=\frac{1}{E_{33}}\left(\tilde{q}_{3}-E_{31} \frac{\partial \tilde{u}_{1}}{\partial x_{1}}-E_{32} \frac{\partial \tilde{u}_{2}}{\partial x_{2}}\right) .
$$

Using expressions (32), (33) from Hooke's Law one may determine the expressions for bimoment intensities $\bar{\sigma}_{11}, \bar{\sigma}_{22}, \bar{\sigma}_{12}, \tilde{\sigma}_{11}, \tilde{\sigma}_{12}, \tilde{\sigma}_{22}$ and $\bar{\sigma}_{11}^{*}, \bar{\sigma}_{22}^{*}, \tilde{\sigma}_{11}^{*}, \tilde{\sigma}_{22}^{*}$. The intensities of bimoments $\bar{\sigma}_{11}, \bar{\sigma}_{22}, \bar{\sigma}_{12}$ are determined in the form:

$$
\begin{gathered}
\bar{\sigma}_{11}=\left(E_{11}-\frac{E_{13}}{E_{33}} E_{31}\right) \frac{\partial \bar{u}_{1}}{\partial x_{1}}+\left(E_{12}-\frac{E_{13}}{E_{33}} E_{32}\right) \frac{\partial \bar{u}_{2}}{\partial x_{2}}+\frac{E_{13}}{E_{33}} \bar{q}_{3}, \\
\bar{\sigma}_{22}=\left(E_{21}-\frac{E_{23}}{E_{33}} E_{31}\right) \frac{\partial \bar{u}_{1}}{\partial x_{1}}+\left(E_{22}-\frac{E_{23}}{E_{33}} E_{32}\right) \frac{\partial \bar{u}_{2}}{\partial x_{2}}+\frac{E_{23}}{E_{33}} \bar{q}_{3}, \\
\bar{\sigma}_{12}=G_{12}\left(\frac{\partial \bar{u}_{1}}{\partial x_{2}}+\frac{\partial \bar{u}_{2}}{\partial x_{1}}\right) .
\end{gathered}
$$

The intensities of bimoments $\tilde{\sigma}_{11}, \tilde{\sigma}_{12}, \tilde{\sigma}_{22}$ are determined by the following formula:

$$
\begin{gathered}
\tilde{\sigma}_{11}=\left(E_{11}-\frac{E_{13}}{E_{33}} E_{31}\right) \frac{\partial \tilde{u}_{1}}{\partial x_{1}}+\left(E_{12}-\frac{E_{13}}{E_{33}} E_{32}\right) \frac{\partial \tilde{u}_{2}}{\partial x_{2}}+\frac{E_{13}}{E_{33}} \tilde{q}_{3}, \\
\tilde{\sigma}_{22}=\left(E_{21}-\frac{E_{23}}{E_{33}} E_{31}\right) \frac{\partial \tilde{u}_{1}}{\partial x_{1}}+\left(E_{22}-\frac{E_{23}}{E_{33}} E_{32}\right) \frac{\partial \tilde{u}_{2}}{\partial x_{2}}+\frac{E_{23}}{E_{33}} \tilde{q}_{3}, \\
\tilde{\sigma}_{12}=G_{12}\left(\frac{\partial \tilde{u}_{1}}{\partial x_{2}}+\frac{\partial \tilde{u}_{2}}{\partial x_{1}}\right) .
\end{gathered}
$$

The intensities of bimoments $\bar{\sigma}_{11}^{*}, \bar{\sigma}_{22}^{*}, \tilde{\sigma}_{11}^{*}, \tilde{\sigma}_{22}^{*}$ have the expressions:

$$
\begin{aligned}
& \bar{\sigma}_{11}^{*}=-E_{11} H \frac{\partial^{2} \bar{W}}{\partial x_{1}^{2}}-E_{12} H \frac{\partial^{2} \bar{W}}{\partial x_{2}^{2}}+E_{11} H \frac{\partial}{\partial x_{1}}\left(\frac{\bar{q}_{1}}{G_{13}}\right)+E_{12} H \frac{\partial}{\partial x_{2}}\left(\frac{\bar{q}_{2}}{G_{23}}\right)+E_{13} \frac{\bar{R}}{H}, \\
& \bar{\sigma}_{22}^{*}=-E_{12} H \frac{\partial^{2} \bar{W}}{\partial x_{1}^{2}}-E_{22} H \frac{\partial^{2} \bar{W}}{\partial x_{2}^{2}}+E_{12} H \frac{\partial}{\partial x_{1}}\left(\frac{\bar{q}_{1}}{G_{13}}\right)+E_{22} H \frac{\partial}{\partial x_{2}}\left(\frac{\bar{q}_{2}}{G_{23}}\right)+E_{23} \frac{\bar{R}}{H}, \\
& \tilde{\sigma}_{11}^{*}=-E_{11} H \frac{\partial^{2} \tilde{W}}{\partial x_{1}^{2}}-E_{12} H \frac{\partial^{2} \tilde{W}}{\partial x_{2}^{2}}+E_{11} H \frac{\partial}{\partial x_{1}}\left(\frac{\tilde{q}_{1}}{G_{13}}\right)+E_{12} H \frac{\partial}{\partial x_{2}}\left(\frac{\tilde{q}_{2}}{G_{23}}\right)+E_{13} \frac{\tilde{R}}{H}, \\
& \tilde{\sigma}_{22}^{*}=-E_{12} H \frac{\partial^{2} \tilde{W}}{\partial x_{1}^{2}}-E_{22} H \frac{\partial^{2} \tilde{W}}{\partial x_{2}^{2}}+E_{12} H \frac{\partial}{\partial x_{1}}\left(\frac{\tilde{q}_{1}}{G_{13}}\right)+E_{22} H \frac{\partial}{\partial x_{2}}\left(\frac{\tilde{q}_{2}}{G_{23}}\right)+E_{23} \frac{\tilde{R}}{H} .
\end{aligned}
$$

Unknown functions $\bar{R}$ and $\tilde{R}$ in expressions (36) and (37) are determined from the system of algebraic equations relative to coefficients of the series (2), obtained from denotations (3) and (16), and presented as

$$
\begin{gathered}
\bar{R}=4 \times\left(2 \times 3 \times A_{3}+4 \times 5 \times A_{5}+\cdots\right)=420(\bar{W}+6 \bar{r}-15 \bar{\gamma}) . \\
\tilde{R}=4 \times\left(1 \times 2 \times A_{2}+3 \times 4 \times A_{4}+\cdots\right)=\tilde{R}=60(3 \tilde{W}+4 \tilde{r}-21 \tilde{\gamma}) .
\end{gathered}
$$

Here are the formulae to determine the displacements on face surfaces of the plate $z=-h$ and $z=+h$ :

$$
u_{i}^{(-)}=\bar{u}_{i}-\tilde{u}_{i}, \quad u_{i}^{(+)}=\bar{u}_{i}+\tilde{u}_{i}, \quad(i=1,2), \quad u_{3}^{(-)}=\tilde{W}-\bar{W}, u_{3}^{(+)}=\tilde{W}+\bar{W} .
$$


Formulae for the stresses on face surfaces of the plate $z=-h$ and $z=+h$ have the form:

$$
\sigma_{i j}^{(-)}=\bar{\sigma}_{i j}-\tilde{\sigma}_{i j}, \quad \sigma_{i j}^{(+)}=\bar{\sigma}_{i j}+\tilde{\sigma}_{i j}, \quad(i=1,2 ; j=1,2) .
$$

Note down the boundary conditions for a cantilever plate. Let the edge of the plate $x_{2}=0$ be rigidly fixed. Remaining edges of the plate are free from supports.

The fixed edge of the plate has zero displacement and the boundary conditions on the edge $x_{2}=0$ are:

$$
\begin{array}{ll}
\tilde{\psi}_{1}=0, & \tilde{\psi}_{2}=0, \quad \tilde{\beta}_{1}=0, \quad \tilde{\beta}_{2}=0, \quad \tilde{r}=0, \quad \tilde{\gamma}=0, \quad \tilde{u}_{1}=0, \quad \tilde{u}_{2}=0, \quad \tilde{W}=0 . \\
\bar{\psi}_{1}=0, & \bar{\psi}_{2}=0, \quad \bar{\beta}_{1}=0, \quad \bar{\beta}_{2}=0, \quad \bar{r}=0, \quad \bar{\gamma}=0, \quad \bar{u}_{1}=0, \quad \bar{u}_{2}=0, \quad \bar{W}=0 .
\end{array}
$$

On the free edge of the plate $x_{2}=b$ boundary conditions are:

$$
\begin{gathered}
M_{12}=0, M_{22}=0, P_{12}=0, \quad P_{12}=0, \quad Q_{23}=0, \quad \tilde{p}_{23}=0, \quad \tilde{\sigma}_{12}=0, \quad \tilde{\sigma}_{22}=0, \quad \tilde{\sigma}_{22}^{*}=0 . \\
N_{11}=0, \quad N_{12}=0, T_{11}=0, \quad T_{12}=0, \quad \bar{\sigma}_{11}=0, \quad \bar{\sigma}_{12}=0, \quad \bar{p}_{13}=0, \quad \bar{\tau}_{13}=0, \quad \bar{\sigma}_{11}^{*}=0 .
\end{gathered}
$$

On two free opposite edges of the plate $x_{1}=0, x_{1}=a$ the following conditions should be fulfilled:

$$
\begin{gathered}
M_{11}=0, M_{12}=0, P_{11}=0, \quad P_{12}=0, \quad Q_{13}=0, \quad \tilde{p}_{13}=0, \quad \tilde{\sigma}_{11}=0, \quad \tilde{\sigma}_{12}=0, \quad \tilde{\sigma}_{11}^{*}=0 . \\
N_{11}=0, \quad N_{12}=0, T_{11}=0, T_{12}=0, \quad \bar{\sigma}_{11}=0, \quad \bar{\sigma}_{12}=0, \quad \bar{p}_{13}=0, \quad \bar{\tau}_{13}=0, \quad \bar{\sigma}_{11}^{*}=0 .
\end{gathered}
$$

On two angular points of the plate, free from supports and external forces, $x_{1}=0, x_{2}=b$ and $x_{1}=a, x_{2}=b$ the following boundary conditions should be fulfilled:

$$
\begin{aligned}
& M_{11}=0, M_{12}=0, P_{11}=0, P_{12}=0, Q_{13}=0, \quad \tilde{p}_{13}=0, \quad \tilde{\sigma}_{11}=0, \quad \tilde{\sigma}_{12}=0, \tilde{\sigma}_{11}^{*}=0 . \\
& N_{11}=0, N_{12}=0, T_{11}=0, T_{12}=0, \bar{\sigma}_{11}=0, \bar{\sigma}_{12}=0, \bar{p}_{13}=0, \bar{\tau}_{13}=0, \bar{\sigma}_{11}^{*}=0 \text {. } \\
& M_{12}=0, M_{22}=0, P_{12}=0, P_{12}=0, Q_{23}=0, \tilde{p}_{23}=0, \tilde{\sigma}_{12}=0, \tilde{\sigma}_{22}=0, \tilde{\sigma}_{22}^{*}=0 \text {. } \\
& N_{11}=0, N_{12}=0, T_{11}=0, T_{12}=0, \bar{\sigma}_{11}=0, \bar{\sigma}_{12}=0, \bar{p}_{13}=0, \bar{\tau}_{13}=0, \bar{\sigma}_{11}^{*}=0 .
\end{aligned}
$$

At initial moment of time $t=0$ initial conditions are taken as zero ones.

The advantage of bimoment theory, when compared to existing ones, is its high accuracy and good applicability to solving practical problems of evaluation of stresses and displacements in orthotropic plates.

\section{Solution of Tests Problem}

Assume that a plate is under the action of external uniformly distributed surface normal load $q_{3}$ on $o z$-axis in the form of Heaviside function applied to face surface of the plate $z=-h$ :

$$
q_{3}= \begin{cases}0, & \text { at } t \leq 0 \\ -q_{0}, & \text { at } t>0,\end{cases}
$$

where $q_{0}$ is a parameter of external force. Remaining components of external forces are zero. 
While obtaining numerical results on displacements, a dimensionless function is introduced:

$$
f=\frac{E_{1} f}{H q_{0}} .
$$

Dimensionless stresses and intensities of bimoments are introduced according to the following formulae:

$$
\sigma_{i j}=\frac{\sigma_{i j}}{q_{0}},(i=1,2 ; j=1,2) .
$$

The problem is solved by the method of finite differences. A finite-difference approximation of displacements derivatives in spatial coordinates is given here. To approximate the internal points of displacements derivatives, the expressions of central difference schemes are used. To approximate the first derivatives one would use the following expressions with respect to the central points

$$
\frac{\partial f_{i, j}^{k}}{\partial x_{1}}=\frac{f_{i+1, j}^{k}-f_{i-1, j}^{k}}{2 \Delta x_{1}}, \quad \frac{\partial f_{i, j}^{k}}{\partial x_{2}}=\frac{f_{i, j+1}^{k}-f_{i, j-1}^{k}}{2 \Delta x_{2}} .
$$

The second displacement derivatives are approximated by the following expressions:

$$
\frac{\partial^{2} f_{i, j}^{k}}{\partial x_{1}^{2}}=\frac{f_{i+1, j}^{k}-2 f_{i, j}^{k}+f_{i-1, j}^{k}}{\Delta x_{1}^{2}}, \quad \frac{\partial^{2} f_{i, j}^{k}}{\partial x_{2}^{2}}=\frac{f_{i, j+1}^{k}-2 f_{i, j}^{k}+f_{i, j-1}^{k}}{\Delta x_{2}^{2}} .
$$

The second derivative with respect to time, using finite-difference equation, is represented in the form:

$$
\frac{\partial^{2} f_{i, j}^{k}}{\partial \tau^{2}}=\frac{f_{i, j}^{k+1}-2 f_{i, j}^{k}+f_{i, j}^{k-1}}{\Delta \tau^{2}}
$$

here $\tau=\frac{c t}{H}$-is a dimensionless time, where $c=\sqrt{\frac{E_{1}}{\rho}}$.

\section{Numeric Results}

Calculations are carried out for square plates with dimensions in plan $a=b=3 H$. Material of the plate is taken as isotropic with elasticity modulus $E_{1}=E_{2}=E_{3}=E_{0}$, shear modulus $G_{12}=G_{13}=G_{23}=E_{0} / 2(1+v)$, Poisson ratio $v_{21}=v_{23}=v_{31}=v=0.3$ and as orthotropic material $15: 1$ with elasticity modulus $E_{1}=4.6 E_{0}, E_{2}=1.6 E_{0}$, shear modulus $G_{12}=0.56 E_{0}, G_{13}=0.43 E_{0}, G_{23}=0.33 E_{0}$, here $E_{0}=10^{4} \mathrm{MPa}$, Poisson ratio $v_{21}=0.27, v_{23}=0.3, v_{31}=0.07$.

Figures 1-3 show the diagrams of changes of dimensionless values of displacements $u_{k}^{(+)}, u_{k}^{(-)},(k=1,3)$ of the points on face surface $z=+h, z=-h$, obtained from the solution of the first and second problems of bimoment theory of plates by formulae (40). The studies have indicated that the form of the bend of generalized displacements $u_{1}^{(+)}, u_{1}^{(-)}$is antisymmetric, and the form of the bend of generalized displacements $u_{i}^{(+)}, u_{i}^{(-)},(i=2,3)$ is symmetric. Maximum dimensionless values of generalized displacements $u_{1}^{(+)}, u_{1}^{(-)}$occur on the limiting points of a free edge of the plate $x_{2}=b$ 
$u_{1}^{(+)}$

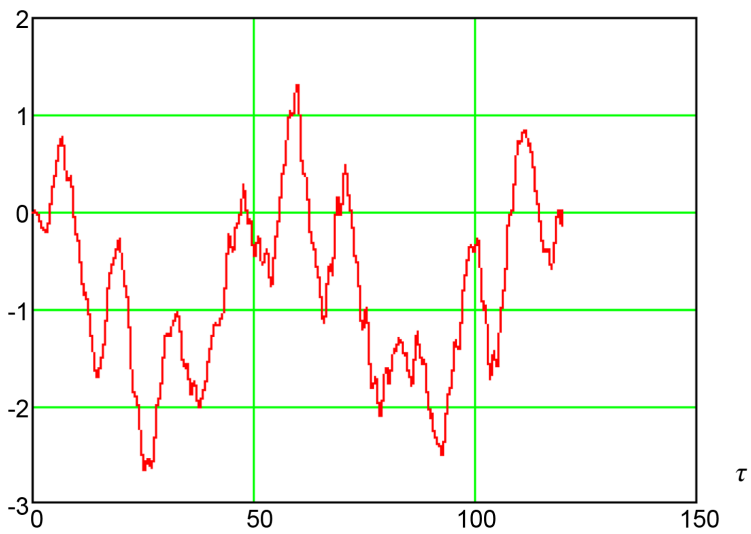

(a)

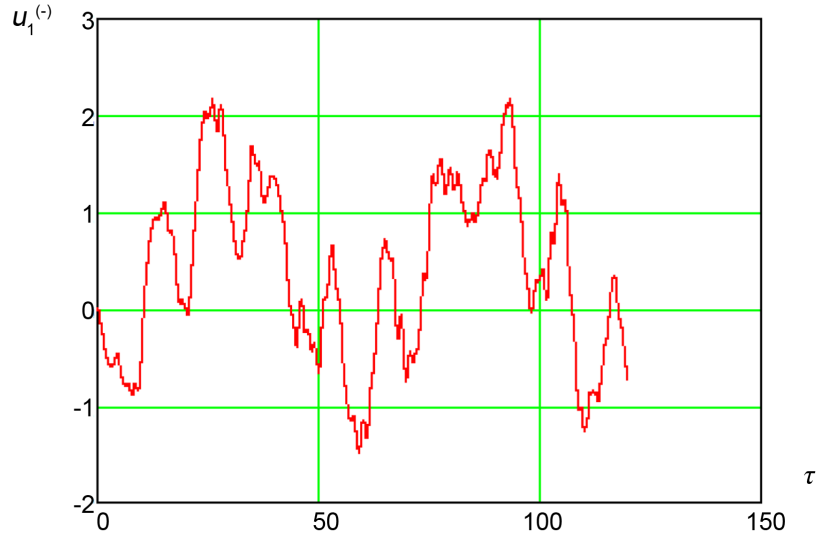

(b)

Figure 1. Diagram of changes in displacements $u_{1}^{(+)}-(\mathrm{a}), u_{1}^{(-)}-(\mathrm{b})$ of the points on face surface of the plate $z=+h, z=-h$ vs time.

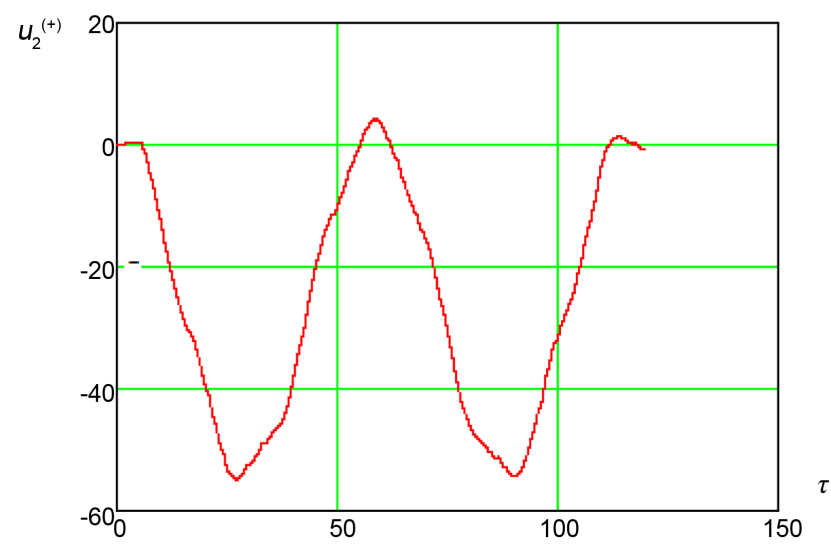

(a)

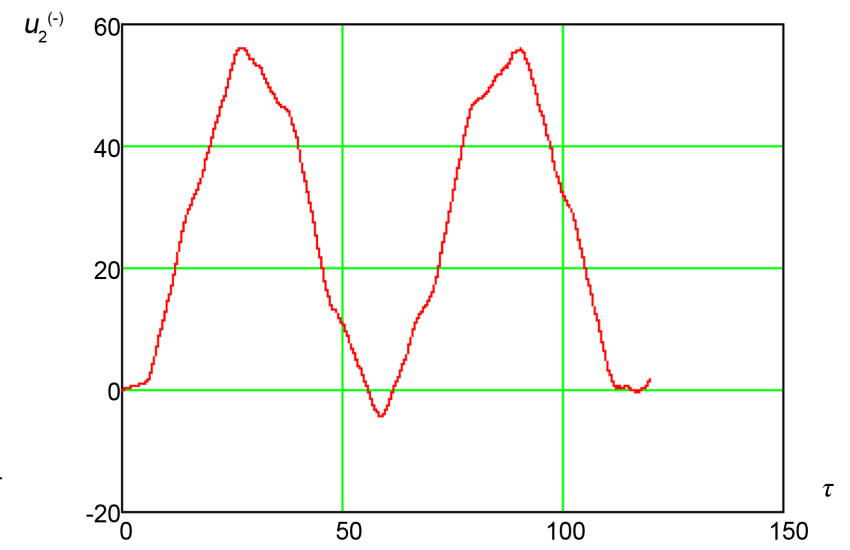

(b)

Figure 2. Diagram of changes in displacements $u_{2}^{(+)}-(\mathrm{a}), u_{2}^{(-)}-(\mathrm{b})$ of the points on face surface of the plate $z=+h, z=-h$ vs time.

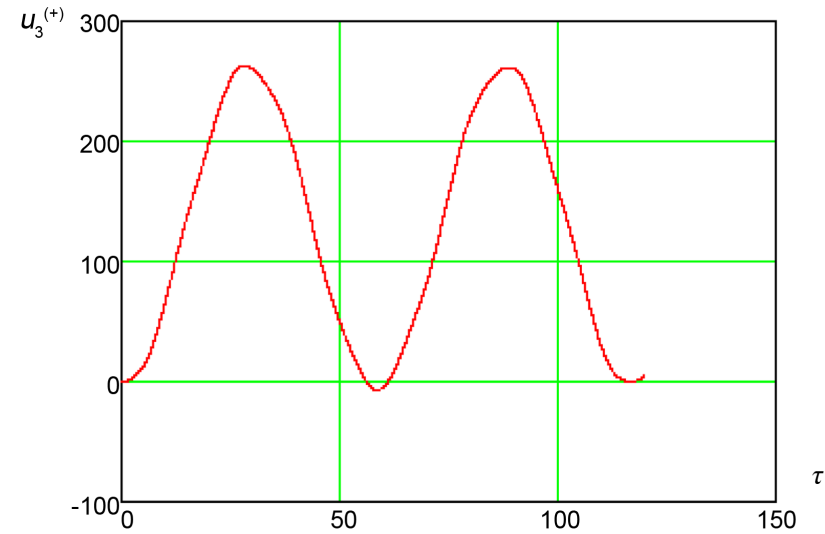

(a)

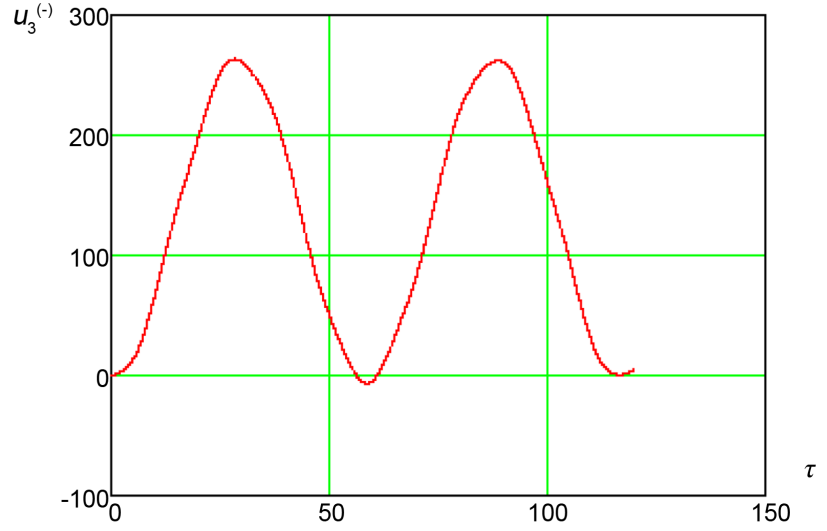

(b)

Figure 3. Diagrams of changes in displacements $u_{3}^{(+)}-(\mathrm{a}), u_{3}^{(-)}-(\mathrm{b})$ of the points on face surface of the plate $z=+h, z=-h$ vs time. 
and have the following values: $u_{1 \max }^{(+)}=-2.673$ (Figure $1(\mathrm{a})$ ) and $u_{1 \max }^{(-)}=2.177$ (Figure $1(\mathrm{~b}))$.

Maximum dimensionless values of displacements $u_{2}^{(+)}, u_{2}^{(-)}$of the points of face surface $z=+h, z=-h$ occur in the middle of a free edge of the plate $x_{2}=b$, they have the following values: $u_{2 \max }^{(+)}=-54.914$ (Figure 2(a)) and $u_{2 \max }^{(-)}=56.004$ (Figure 2(b)).

Maximum dimensionless values of displacements $u_{3}^{(+)}, u_{3}^{(-)}$of the points on face surface $z=+h, z=-h$ occur in the middle of a free edge of the plate $x_{2}=b$ and have the following values: $u_{3 \max }^{(+)}=262.936$ (Figure 3(a)) and $u_{3 \max }^{(+)}=263.422$ (Figure 3(b)).

Figure 4 and Figure 5 indicate the diagrams of changes in dimensionless values of normal stresses of the points on face surface of the plate $z=+h, z=-h$, obtained by formulae (41) from the solutions of the first and second problems of bimoment theory of plates. Maximum dimensionless values occur in the middle of a fixed edge of the plate $x_{2}=b$ and have the following values $\sigma_{11}^{(+)}=-24.968$ (Figure 4(a)), $\sigma_{11}^{(-)}=25.570$ (Figure 4(b)).

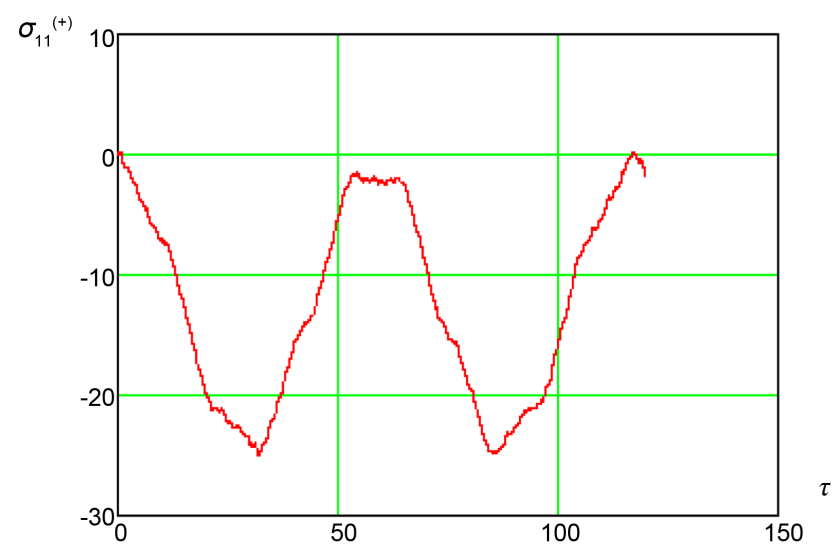

(a)

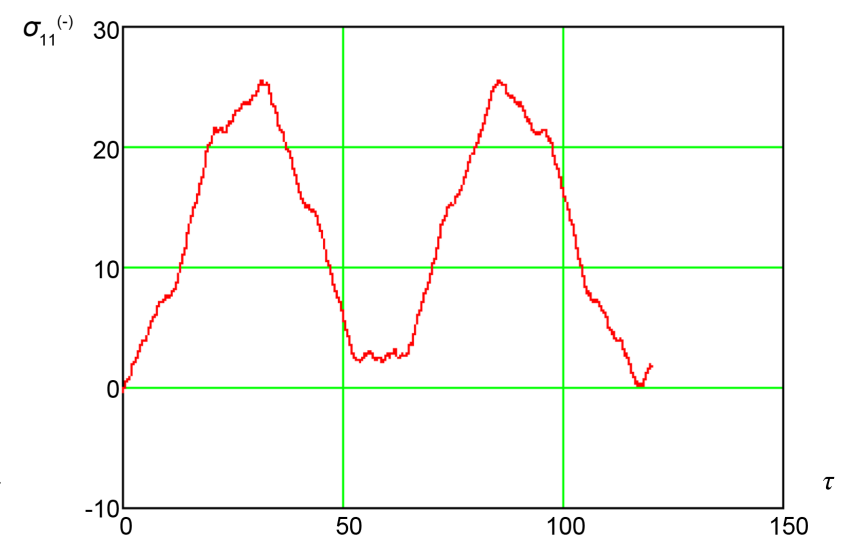

(b)

Figure 4. Diagrams of changes in stresses $\sigma_{11}^{(+)}-(\mathrm{a}), \sigma_{11}^{(-)}-(\mathrm{b})$ of the points on face surface of the plate $z=+h, z=-h \quad$ vs time.

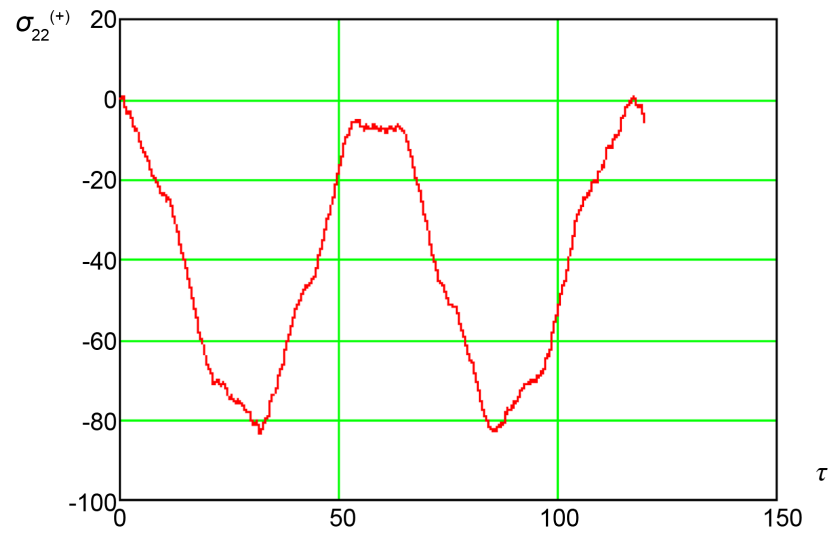

(a)

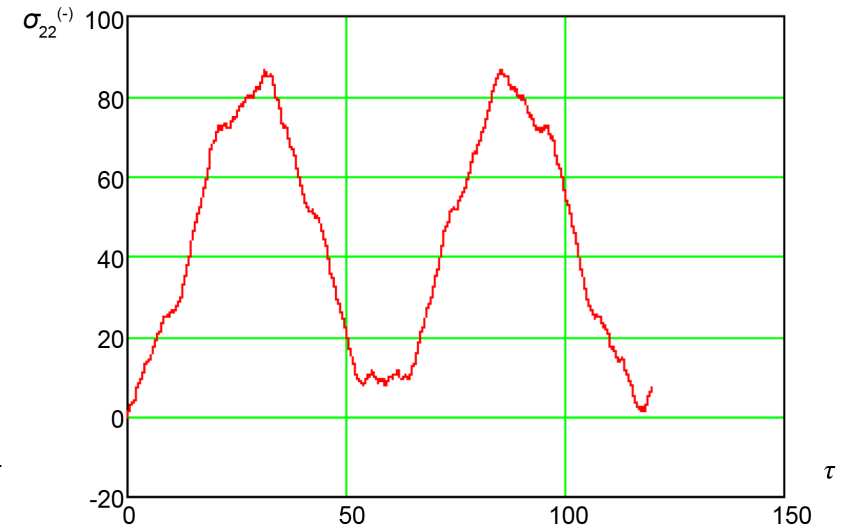

(b)

Figure 5. Diagram of changes in stresses $\sigma_{22}^{(+)}-(\mathrm{a}), \sigma_{22}^{(-)}-(\mathrm{b})$ of the points on face surface of the plate $z=+h, z=-h$ vs time. 
Maximum dimensionless values of stresses $\sigma_{22}^{(+)}, \sigma_{22}^{(-)}$of the points on face surface of the plate $z=+h, z=-h$ occur in the middle of the fixed edge of the plate $x_{2}=b$. Maximum dimensionless values have the following values: $\sigma_{22}^{(+)}=-83.371$ (Figure $5(\mathrm{a})$ ) and $\sigma_{22}^{(-)}=86.412$ (Figure 5(b)). As could be seen, numerical values of displacements and stresses $u_{2}, \sigma_{22}$ are substantially greater than numerical values of displacements and stresses $u_{1}, \sigma_{11}$ in the same observed points on face surface of the plate.

Figure 6 and Figure 7 indicate the diagrams of changes in dimensionless values of normal stresses on face surface of orthotropic plate $z=+h, z=-h$, obtained from the solutions of the first and second problems of bimoment theory of plates by formulae (41). Maximum dimensionless values occur in the middle of a fixed edge of orthotropic plate $x_{2}=b$ and have the following values $\sigma_{11}^{(+)}=-23.599$ (Figure 6(a)) and $\sigma_{11}^{(-)}=24.278$ (Figure 6(b)). Maximum dimensionless stresses $\sigma_{22}^{(+)}, \sigma_{22}^{(-)}$of the points on face surface $z=+h, z=-h$ of orthotropic plate occur in the middle of a fixed edge

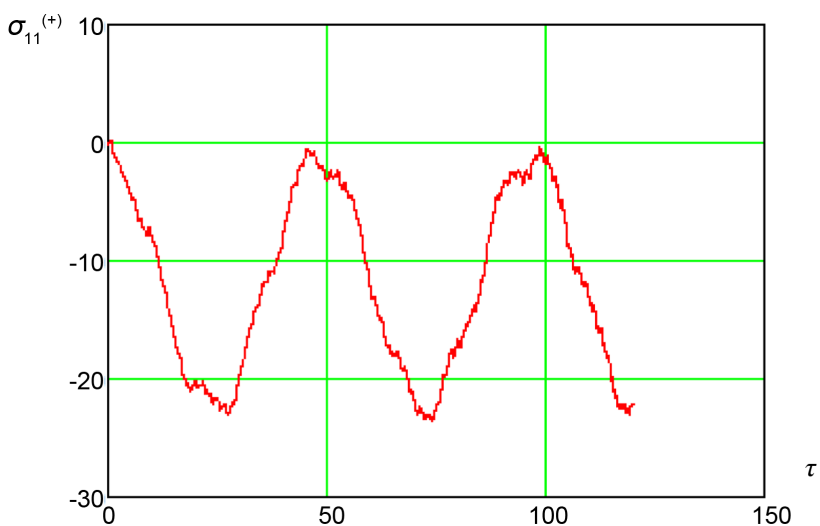

(a)

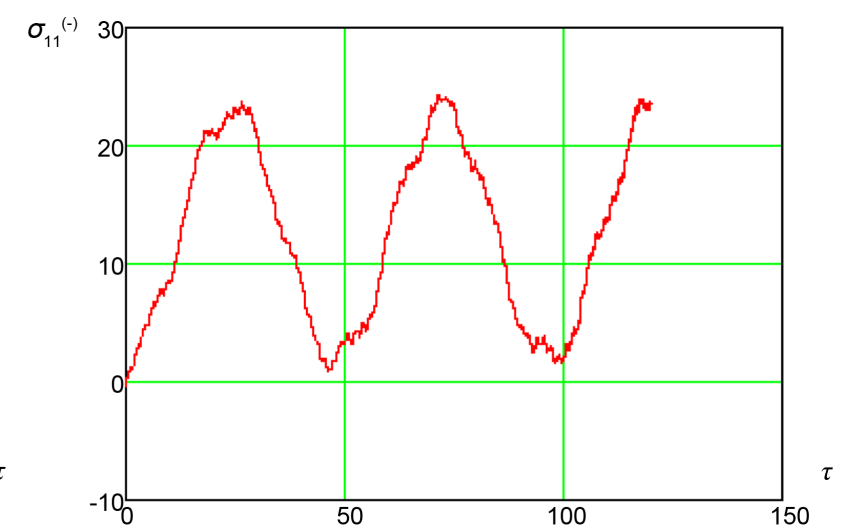

(b)

Figure 6. Diagrams of changes in stresses $\sigma_{11}^{(+)}$-(a), $\sigma_{11}^{(-)}$-(b) of the points on face surface of orthotropic plate $z=+h, z=-h$ vs time.

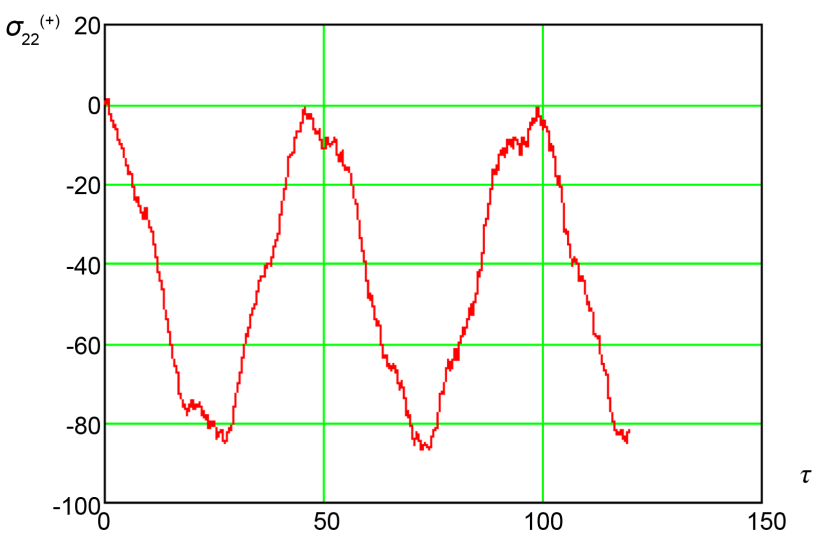

(a)

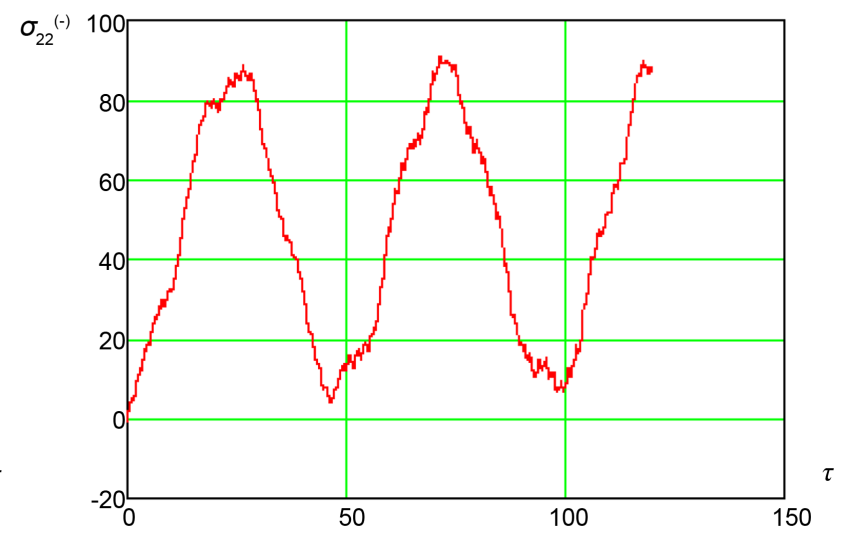

(b)

Figure 7. Diagrams of changes in stresses $\sigma_{22}^{(+)}-(\mathrm{a}), \sigma_{22}^{(-)}-(\mathrm{b})$ of the points on face surface of orthotropic plate $z=+h, z=-h$ vs time. 
of the plate $x_{2}=b$. Maximum dimensionless values are $\sigma_{22}^{(+)}=-86.681$ (Figure 7(a)) and $\sigma_{22}^{(-)}=90.919$ (Figure $7(\mathrm{~b})$ ).

If to solve this problem by Timoshenko theory, the maximum stresses for isotropic plates equal to $\sigma_{11}=-16.531, \sigma_{22}=-55.183$, and for orthotropic plate equal to $\sigma_{11}=-14.125, \sigma_{22}=-52.485$. As seen, numerical values of stresses, obtained by Timoshenko theory are considerably less compared to bimoment theory of plates.

The laws of changes of generalized displacements and force factors in time for the first and second problems are identical to the laws of displacement changes in time, presented in Figures 1-7. Further consider only maximum values of generalized displacements, forces, moments, and bimoments obtained from the solution of the first and second problems. Tables 1-4 show maximum values of kinematic and force factors of the problems.

Table 1 and Table 2 show dimensionless numerical results of kinematic functions calculation for isotropic and orthotropic plate, obtained from the solution of the second problem.

Table 3 gives numerical results of calculation of dimensionless longitudinal forces $n_{22}=\frac{N_{22}}{H}, n_{12}=\frac{N_{12}}{H}$ and bimoments $\frac{\bar{\sigma}_{11}}{q_{0}}, \frac{\bar{\sigma}_{22}}{q_{0}}, \frac{\bar{p}_{23}}{q_{0}}, \frac{\bar{p}_{33}}{q_{0}}, t_{11}=\frac{T_{11}}{H q_{0}}, t_{22}=\frac{T_{22}}{H q_{0}}$. As could be seen, the values of forces and bimoments of the plate $N_{22}, T_{22}, \bar{p}_{33}, \bar{p}_{23}$ are commensurable, and the values of bimoments $\bar{\sigma}_{11}, \bar{\sigma}_{22}$ are substantially greater than the

Table 1. The values of kinematic functions of the first problem.

\begin{tabular}{ccccccc}
\hline Material & $E_{1} \bar{u}_{1} / H q_{0}$ & $E_{1} \bar{u}_{2} / H q_{0}$ & $E_{1} \bar{\psi}_{2} / H q_{0}$ & $E_{1} \bar{\beta}_{2} / H q_{0}$ & $E_{1} \bar{r} / H q_{0}$ & $E_{1} \bar{W} / H q_{0}$ \\
\hline isotropic & \pm 0.562 & 1.089 & 1.029 & 0.350 & -0.253 & -0.694 \\
orthotropic & \pm 0.155 & 0.983 & 0.817 & 0.289 & -0.221 & -0.573 \\
\hline
\end{tabular}

Table 2. The values of kinematic functions of the second problem.

\begin{tabular}{ccccccc}
\hline Material & $E_{1} \tilde{u}_{1} / H q_{0}$ & $E_{1} \tilde{u}_{2} / H q_{0}$ & $E_{1} \tilde{\psi}_{2} / H q_{0}$ & $E_{1} \tilde{\beta}_{2} / H q_{0}$ & $E_{1} \tilde{r} / H q_{0}$ & $E_{1} \tilde{W} / H q_{0}$ \\
\hline isotropic & \pm 2.368 & -55.457 & 18.432 & -11.070 & 263.081 & 263.173 \\
orthotropic & \pm 2.391 & -36.789 & 12.229 & -7.344 & 179.450 & 179.557 \\
\hline
\end{tabular}

Table 3. The values of longitudinal forces and bimoments of the first problem.

\begin{tabular}{ccccccc}
\hline Material & $\bar{\sigma}_{11} / q_{0}$ & $\bar{\sigma}_{22} / q_{0}$ & $t_{22} / q_{0}$ & $n_{22} / q_{0}$ & $\bar{p}_{33} / q_{0}$ & $\bar{p}_{23} / q_{0}$ \\
\hline isotropic & 0.765 & 3.081 & 0.266 & -0.654 & -0.300 & -0.199 \\
orthotropic & 0.998 & 4.254 & 0.330 & -0.776 & -0.280 & -0.173 \\
\hline
\end{tabular}

Table 4. The values of moments, bimoments and shear forces of the second problem.

\begin{tabular}{ccccccc}
\hline Material & $\tilde{\sigma}_{11} / q_{0}$ & $\tilde{\sigma}_{22} / q_{0}$ & $m_{22} / q_{0}$ & $p_{22} / q_{0}$ & $\tilde{p}_{33} / H q_{0}$ & $Q_{23} / H q_{0}$ \\
\hline isotropic & -5.174 & -84.492 & -9.193 & -5.954 & -4.852 & 5.733 \\
orthotropic & -23.679 & -87.882 & -9.019 & -5.818 & -3.431 & 5.752 \\
\hline
\end{tabular}


values of remaining bimoments.

Table 4 presents dimensionless numerical results of calculation of bending moments, forces and bimoments $\tilde{\sigma}_{11}, \tilde{\sigma}_{22}, m_{22}=\frac{2 M_{22}}{H^{2}}$, longitudinal bending bimoments $\tilde{p}_{33}, p_{22}=\frac{2 P_{22}}{H^{2}}$ and shear force $Q_{23} / H q_{0}$. Similarly, numerical values of forces and bimoments are commensurable, and the values of bimoments $\tilde{\sigma}_{11}, \tilde{\sigma}_{22}$ are many times greater than the values of remaining forces and bimoments.

A step in calculation on dimensionless coordinates is taken as $\Delta x_{1}=\frac{a}{60}, \Delta x_{1}=\frac{b}{60}$. The stability of iteration in dimensionless time is provided by explicit scheme with $\Delta \tau=0.02$ step.

According to the analysis of results shown in Tables 1-4, the following conclusions can be drawn: numerical values of kinematic functions and force factors (Table 1 and Table 3), obtained by solving the first problem, characterize the tension-compression in longitudinal direction, taking into account the transverse reduction of the plate; numerical values of kinematic functions and force factors (Table 2 and Table 4), obtained by solving the second problem, characterize the lateral bending with consideration of transverse shear of the plate. Comparing the numerical results of the first and second problems, it could be noted that the numerical values of displacements and force factors in the second problem is much greater than the corresponding displacement values and force factors of the first problem.

\section{Conclusions}

Technique of constructing a bimoment theory of the plate, which takes into account the forces, moments and bimoments, developed by nonlinear law of displacements distribution in cross-sections of the plate is briefly presented here. Exact expressions of internal forces, moments and bimoments are given, as well as the equations of motion and boundary conditions for orthotropic thick plate.

Bimoment theory of the plate is applied to solving the dynamic problem of forced oscillations of orthotropic thick plate. An example of forced oscillations of cantilever plate under the influence of transverse dynamic loads in the form of the Heaviside function is considered. Based on the method of finite differences, the methods for calculating the dynamic cantilever plate are developed. Numerical results of displacements, forces, moments, bimoments and stresses for cantilever plate are obtained and followed by analysis. Based on the analysis of numerical results, a conclusion is drawn that Timoshenko theory is not acceptable for the calculation of displacements and stresses of the plate under dynamic effects.

\section{References}

[1] Ambartsumyan, S.A. (1987) Theory of Anisotropic Plates. Nauka, Moscow, 360 p.

[2] Galimov, K.Z. (1977) Theory of Shells with Account of Transverse Shear. Kazan University, Kazan, 212 p. 
[3] Galimov, Sh.K. (1976) Specified Theory of Calculation of Orthotropic Rectangular Plate under Lateral Load. Investigations in Theory of Plates and Shells, SAT Articles, Kazan, Vol. XII, 78-84.

[4] Mushtari, Kh.M. (1990) Nonlinear Theory of Shells. Nauka, Moscow, 223 p.

[5] Vlasov, B.F. (1952) On a Case of Bending of a Rectangular Thick Plate Vestnik MGU Mechanics. Mathematics, Astronomy and Chemistry, No. 2, 25-34.

[6] Baida, E.N. (1983) Some Spatial Problems of Elasticity. Leningrad University, Leningrad, $232 \mathrm{p}$.

[7] Karamooz Ravari, M.R. and Forouzan, M.R. (2011) Frequency Equations for the In-Plane Vibration of Orthotropic Circular Annular Plate. Archive of Applied Mechanics, 81, 1307 1322. http://dx.doi.org/10.1007/s00419-010-0488-6

[8] Chang, H.-H. and Tarn, J.-Q. (2012) Three-Dimensional Elasticity Solutions for Rectangular Orthotropic Plates. Journal of Elasticity, 108, 49-66. http://dx.doi.org/10.1007/s10659-011-9355-0

[9] Zenkour, A.M., Allam, M.N.M., Shaker, M.O. and Radwan, A.F. (2011) On the Simple and Mixed First-Order Theories for Plates Resting on Elastic Foundations. Acta Mechanica, 220, 33-46. http://dx.doi.org/10.1007/s00707-011-0453-7

[10] Akhmedov, A.B. (2007) Comparative Analysis of Specified Theories of Bending of Thick Plates. Uzbek Journal "Problems of Mechanics", No. 2, 44-46.

[11] Akhmedov, A.B. (2007) Dynamic Effect of Concentrated Forces in Thick Plates. Uzbek Journal "Problems of Mechanics", No. 4, 31-36.

[12] Usarov, M.K. (2014) Calculation of Orthotropic Plates Based on the Theory of Bimoments. Uzbek Journal Problems of Mechanics, No. 3-4, 37-41.

[13] Usarov, M.K. (2014) Bimoment Theory of Bending and Vibrations of Orthotropic Thick Plates. Vestnik NUU, No. 2/1, 127-132.

[14] Usarov, M.K. (2015) Bending of Orthotropic Plates with Consideration of Bimoments. Civil Engineering Journal, No. 1, 80-90.

[15] Usarov, M.K. (2015) On Solution of the Problem of Bending of Orthotropic Plates on the Basis of Bimoment Theory. Open Journal of Applied Sciences, 5, 212-219. http://dx.doi.org/10.4236/ojapps.2015.55021

[16] Usarov M.K. (2015) Bending of Orthotropic Plates with Consideration of Bimoments. Civil Engineering Journal, No. 1, 80-90.

[17] Usarov M.K. (2015) On Solution of the Problem of Bending of Orthotropic Plates on the Basis of Bimoment Theory. Open Journal of Applied Sciences, 5, 212-219. http://dx.doi.org/10.4236/ojapps.2015.55021 
Submit or recommend next manuscript to SCIRP and we will provide best service for you:

Accepting pre-submission inquiries through Email, Facebook, LinkedIn, Twitter, etc. A wide selection of journals (inclusive of 9 subjects, more than 200 journals)

Providing 24-hour high-quality service

User-friendly online submission system

Fair and swift peer-review system

Efficient typesetting and proofreading procedure

Display of the result of downloads and visits, as well as the number of cited articles

Maximum dissemination of your research work

Submit your manuscript at: http://papersubmission.scirp.org/

Or contactwjm@scirp.org 